\section{Chemistry of metal complexes}

Metal Complexes in Organic Chemistry. By R.P. Houghton. Pp.308. (Cambridge University Press: Cambridge, 1979.) Hardback $£ 21$; paperback $£ 7.50$.

I ENJOYED reading this book; it is written in a concise manner and is a mine of useful information. Its complex chemistry is a little thin in places, but perhaps the dose is correctly adjusted to the organic chemists whom the book addresses. The author has done a remarkably fine job in bringing together and systematising the vast variety of interesting organic reactions made possible or more specific, catalysed, inhibited or altered in direction by the action of metal containing compounds.

The book is aimed at "advanced undergraduates, graduates at the start of a research career in organic chemistry and practising organic chemists who wish to learn more about the chemistry of metal complexes"'. It is an excellent book for that purpose, and must represent an enormous amount of work on the part of its author. Its main deficiency is a lack of reference to recent primary literature, obviously a matter of policy, but it does give reference to some 125 well chosen reviews.

In 308 pages it is quite impossible to catalogue all of the organic reactions mediated by metal complexes. Nevertheless the author has made a very comprehensive survey of reaction types -except olefin polymerisation, but Ziegler polymerisation of olefins does receive brief mention (page 244). Each type of organic reaction is profusely illustrated by examples, well set out in display formulae. This means that a mere inorganic chemist such as your reviewer was never at a loss to understand the nature of the organic materials mentioned in the text, even in the very few cases where the name did not convey to him the essential chemical structure intended - for example, "methylmagnesium carbonate" on page 104 is not the probably hypothetical MeMgO.CO.OMgMe, but methyl methoxymagnesium carbonate, $\mathrm{MeOMgO}$.CO.OMe.

The first and second chapters are concerned with general principles of complex chemistry in their application to the explanation of organic reactions mediated by metal complexes. They are very useful chapters with emphasis on electronic effects relating mainly to $\sigma$ - and $\pi$-donation by ligands, $\pi$-donation by the metal atom, relative metal-ligand affinities, steric effects, and coordination saturation. They indicate how these affect the egress of organic reagents to the metal, whether as strongly or weakly bound ligands, and how the reagents are polarised or otherwise activated to chemical attack. The rationalisation of the complex chemistry which is desired for the purpose of this book is good. It is only deficient in taking no account of ligand field theory, especially to explain the stabilisation of the higher valent states of such metals as iron, cobalt and nickel, for example, by thio and non- $\pi$-donor ligands (page 47) and in the explanation of differences between the light and the heavier transition metals. Some short outline of ligand field effects, with a reference to appropriate reviews by the late Sir Ronald Nyholm or L.E. Orgel would have been appropriate here.

The types of reactions covered in the text are much too numerous to mention in this review. They range from the venerable Fredel-Crafts alkylations catalysed by aluminium chloride to the recently developed homogeneous hydrogenation, carbonylation and decarbonylation catalyses based on the heavier Group VIII metal complexes with tertiary phosphines, carbon monoxide (and so on) as ligands. The use of metal complexes in organic chemical synthesis ranges from the simple separation and purification of organic products as ligands, through mediation in organic reactions by shifting equilibria in favour of the desired product, through ester hydrolyses, bond cleavages especially of the $\mathrm{CH}$ bond, subsititutions, isomerisations especially of alkenes,

\section{Neurophysiological offerings}

Studies in Neurophysiology. Presented to A.K. McIntryre. Edited by R. Porter. Pp.440. (Cambridge University Press: Cambridge and New York, 1979.) £32.50.

THIS book, as it proclaims on its cover, is a collection of offerings from his former collaborators and students to a muchloved and respected Australian neurophysiologist, Professor A.K. McIntyre. Professor McIntyre was successively head of the Department of Neurophysiology at Otago and the Department of Physiology at Monash, which quickly grew into a hugely successful venture. The success of these two departments can quickly be appreciated by a glance down the star-spangled list of contributors to this book, many of whose names would be recognised (with enthusiasm, one hopes) by any undergraduate student of neurophysiology.

As an offering to Professor McIntyre, it must have given great pleasure; but how does it rate as a book about neurophysiology? The main difficulty in compiling such a book for publication as opposed to presentation is obviously to keep a coherent thread running through it, and to achieve consistency of style among the many contributions. Many of the hydrogenations, additions and eliminations of various sorts. They are all there, with a strong emphasis on explanations in terms of established physical-organic chemical mechanisms. These are clearly set out and copiously illustrated.

Although I sense that the author lacks some feel for inorganic chemistry, for example, as in his assertion that aluminium chloride exists as $\mathrm{Al}_{2} \mathrm{Cl}_{6}$ molecules in the solid state (page 127) and his formulation of $\left[\mathrm{ReCl}_{3}\right.$ (NMe) $\left.\left(\mathrm{PEt}_{2} \mathrm{Ph}\right)_{2}\right]$ with a nonbonding electron pair on the nitrogen atom (page 96), the book is a text which I strongly recommend. It is well researched, organised and printed. It summarises beautifully the types of interesting organic reactions which occur on metal complexes and has a very useful 10-page index. At present-day prices it is good value at £21. I congratulate the author and his publisher on a unique and well produced book in an area of chemistry which has been surprisingly slow in catching the imagination of organic chemists.

\section{Joseph Chatt}

Joseph Chatt is Director of the ARC Unit of Nitrogen Fixation and Professor of Chemistry at the University of Sussex, Brighton, UK.

individual contributions are, as one would expect, excellent. There are, for example, clear and elegant reviews of such topics as the vesicle hypothesis (Katz), the mechanism of excitation of carotid body chemoreceptors (Eyzaguirre \& Fidone) and the functional significance of changes in extracellular potassium concentration in the central nervous system (Somjen). Other authors have chosen to give much more specialised and technical accounts of particular research projects (for example, Laporte on muscle spindle innervation, and Mubbard on the effects of angiotensin on brain neurones).

Towards the end another abrupt gear change takes place with a long and heady philosophical article by Trevarthen called 'Self-organising systems in psychobiology' (Typical headings: Is a mind biology possible?; Anatomies of imagery and intention; Normal duplicity of brains) which is followed by an essay on Claude Bernard and the milieu interieur.

Though good in places, without a doubt, this collection amounts overall to an indigestible motley which might have been better kept as a private retirement present than expensively dressed up for publication. Unless you are in a sentimental mood, the outlay would be hard to justify.

H.P. Rang

H.P. Rang is Professor of Pharmacology at University College, London, UK. 